\title{
In-vivo Strain and Stress Estimation of the Left Ventricle from MRI Images
}

\author{
Zhenhua $\mathrm{Hu}^{1}$, Dimitris Metaxas², and Leon $\mathrm{Axel}^{3}$ \\ ${ }^{1}$ Department of Computer and Information Science \\ University of Pennsylvania, Philadelphia, PA 19104, USA \\ zhhu@seas.upenn.edu \\ 2 The Center of Computational Biomedical, Imaging and Modeling \\ Rutgers University, New Brunswick, NJ 08854-8019, USA \\ dnm@cs.rutgers.edu \\ ${ }^{3}$ Department of Radiology \\ New York University, New York City, NY 10016, USA \\ Leon.axel@rad.nyu.edu
}

\begin{abstract}
Little information is known about in-vivo heart strain and stress distribution. In this paper, we present a novel statistical model to estimate the invivo material properties and strain and stress distribution in the left ventricle. The displacements of the heart wall are reconstructed in previous work of our group by using MRI-SPAMM tagging technique and deformable model. Based on the reconstructed displacements, we developed the statistical model to estimate strain and stress by using EM algorithm. Two normal hearts and two hearts with right-ventricular hypertrophy are studied. We find noticeable differences in the strain and stress estimated for normal and abnormal hearts.
\end{abstract}

\section{Introduction}

To better understand cardiac diseases, we need to get more information about cardiac motion. Among the factors characterizing heart's motion, stress and strain are two of the most important determinants of various aspects of cardiac physiology and pathophysiology [1]. Stress and strain in the heart depends on not only the structure and material properties of heart but also the active force generated by the heart muscle and the blood pressure on the heart wall. To fully understand it, we need clear pictures on all these issues. The structure of heart has been intensively investigated in the last four decades. It's known that myocardium consists of locally parallel muscle fibers, a complex vascular network, and a dense plexus of connective tissue[1]. Systematic measurements of muscle fiber orientations were carried out by Streeter [10]. His main finding was that fiber directions generally vary in a continuous manner from $+60^{\circ}$ on the endocardium to $-60^{\circ}$ on the epicardium. The material properties of heart wall were initially based on uniaxial tests mostly performed with papillary muscles $[17,18]$. Then the biaxial tests were carried out $[19,20]$ and the constitutive relations were proposed [21,22], which has shown that the myocardium is anisotropic. The active force was modeled in [13] and the blood pressure can be referred in [23]. In the last few years, some experiments were carried out on in-vitro left ventricle strain and stress [2]. But little in-vivo cardiac stress and strain has been estimated. This is because any invasive method will change the heart's material property and non-invasive method is not yet possible to measure these parameters. Some other experiments estimated in- 
vivo strain by using echocardiography [5,24]. But echocardio-graphy doesn't have accurate spatial resolution compared to MRI.

In this paper, the displacements of the left ventricle are reconstructed by using MRI-SPAMM tagging [3] and physics-based deformable model [4] based on previous work of Haber [14,25]. First we present a novel statistical model to estimate the material properties of left ventricle. Second we use the statistical model to compute the strain and stress distribution in different time intervals between the end of diastole and the end of systole.

\section{Mechanical Model}

\subsection{Geometrical Model}

The geometrical model is chosen the same as given in [14,25]. We added the fiber direction into each element. The left ventricle is divided into two layers circumferentially, we assume the fiber is $+60^{\circ}$ on the endocardium and $-60^{\circ}$ on the epicardium.

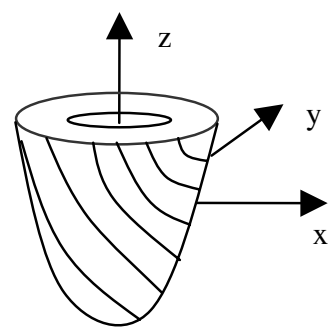

Fig. 1. Fiber orientation on epicardium of left ventricle

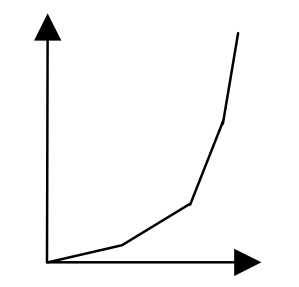

Fig. 2. Piece-wise linearity of Young's modulus $E_{1}$ and $E_{2}$

\subsection{Strain-Stress Relationship}

Myocyte is the main structural components of myocardium, which normally occupies $70 \%$ of ventricular wall volume. Myocytes look like cylinders and are embedded in a complex matrix of collagen, elastin, etc [1]. In recent work [5,6], transversely isotropic linear material model were used in the estimation of cardiac motion. Unlike in $[5,6]$, we model the myocardium as transversely isotropic material with symmetric stiffness matrix and piece-wise linear elasticity. The stress-strain relationship is given by [8]:

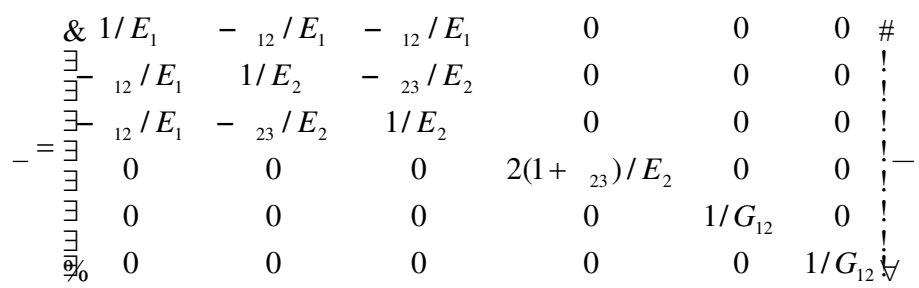


where $E_{1}$ is the Young's modulus along fiber direction, $E_{2}$ is the Young's modulus along cross-fiber direction, $v_{12}$ and $v_{23}$ are the corresponding Poisson ratios, and $G_{12}$ is the Shear Modulus. Since the myocardium is approximately incompressible, the Poisson ratios are both set to 0.4 [9]. Both Young's modulus are assumed piece-wise linear, which are shown qualitatively in Fig 2.

\subsection{Coordinates Transformation Relation}

Since the myofiber direction varies in different parts of the left ventricle [10], we need to transform the local fiber coordinates into global element coordinate when implementing finite element method. For coordinates shown in Fig. 3, the stress in local fiber coordinate $(1,2,3)$ can be transformation into global element coordinate $(x, y, z)$ by [8]:

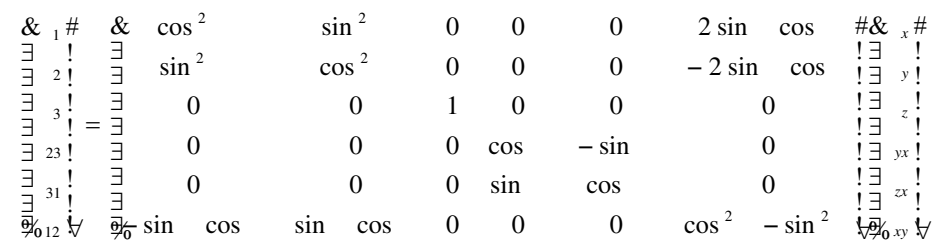

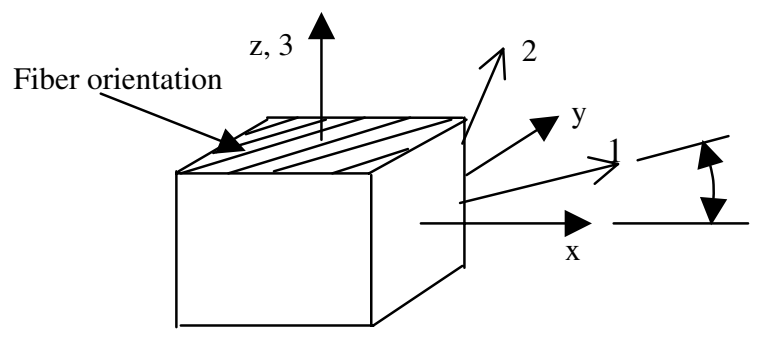

Fig. 3. Coordinate transformation between local and global coordinates

\subsection{Model Dynamics}

Using the energy minimization and variational formulation [11], we derived the finite element equation:

$$
\dot{q}+K q=P
$$

where $q$ represents the displacement, $K$ is the stiffness matrix and:

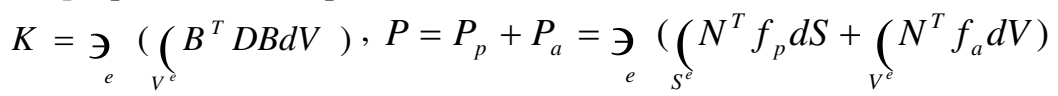

where $f_{p}$ is the boundary force mainly generated by the blood, and $f_{a}$ is the active force generated by the myofiber. $D$ is the stress-strain matrix that relates nodal stress, $\sigma$, to nodal strain, $\varepsilon$, as $\sigma=D \varepsilon$. $B$ is the strain-displacement matrix that relates nodal strain, $\varepsilon$, to nodal displacements, $q$, as $\varepsilon=B q$. $N$ is the shape function for interpolation. 


\section{Statistical Estimation of $K$ and $f_{a}$}

In equation 3, stiffness matrix $K$ and active force $f_{a}$ are unknown. We need to estimate them before the calculation of strain and stress. The in-vitro stiffness parameters were measured through experiments carried on canine heart [13], we'll use the data as the initial state based on similarity between human and canine hearts. The Expectation/Maximization (EM) algorithm is used for the estimation.

\subsection{Expectation/Maximization (EM) Algorithm}

EM algorithm [15] is typically used to compute maximum likelihood estimates given incomplete samples. Define $\mathrm{J}\left(\theta \mid \theta_{0}\right)$ as:

$$
J\left(\theta \mid \theta_{0}\right) \equiv E_{\theta_{0}} ; \log \frac{p(X, \theta)}{p\left(X, \theta_{0}\right)} \mid S(X)=s{\underset{*}{*}}_{*}^{+}
$$

where $\mathrm{X}$ is the random variable, $\theta$ is the parameter to be estimated, $\mathrm{S}(\mathrm{X})$ is the sufficient statistics on $\mathrm{X}, \mathrm{p}(\mathrm{X}, \theta)$ is the probability density function. The EM algorithm works as following:

1. Initialize $\theta_{\text {old }}=\theta_{0}$

2. Compute $\mathrm{J}\left(\theta \mid \theta_{\text {old }}\right)$ for as many values of $\theta$ as possible

3. Maximize $\mathrm{J}\left(\theta \mid \theta_{\text {old }}\right)$ as a function of $\theta$

4. Set $\theta_{\text {new }}=\arg \max \mathrm{J}\left(\theta \mid \theta_{\text {old }}\right)$, if $\theta_{\text {old }} \neq \theta_{\text {new }}$, set $\theta_{\text {old }}=\theta_{\text {new }}$ and go to step 2, otherwise, return $\theta=\theta_{\text {new }}$.

where Step 2 is often referred to as the expectation step and Step 3 is called the maximization step.

\subsection{Implementation}

In our experiment, $\theta=\left(\mathrm{E}_{1}, \mathrm{E}_{2}, f_{a}\right)$. Since we don't have explicit form of probability density function $\mathrm{p}(\mathrm{X}, \theta)$, we use the following way to construct it. We define the displacement divergence as:

$$
d(x, \hat{\theta})=\frac{1}{n} \ni_{i=1}^{n}\left[\left(x_{i}-x_{i t}\right)^{2}+\left(y_{i}-y_{i t}\right)^{2}+\left(z_{i}-z_{i t}\right)^{2}\right]
$$

where $\left(x_{i}, y_{i}, z_{i}\right)$ is the computed displacement based on estimation $\theta=\hat{\theta}$ and $\left(x_{i p}, y_{i t}, z_{i t}\right)$ is the reconstructed displacement from MRI tagging. Since the smaller the displacement divergence, the better the estimation is, we put more weight on estimation with less displacement divergence. Then the normalized density function is defined as:

$$
p(x, \hat{\theta})=\frac{1 / d(x, \hat{\theta})}{\ni_{\theta \in \Theta}(1 / d(x, \theta))}
$$

As shown in Fig. 2, $E_{1}$ and $E_{2}$ are stepwise linear, so we need to estimate $\theta$ in each time interval. We assume $\mathrm{E}_{1}$ and $\mathrm{E}_{2}$ are linearly related. The implementation algorithm is:

1. Initialize $\mathrm{t}=1$

2. In the $t$ th time interval, calculate $\mathrm{d}(\theta)$ and $\mathrm{p}(\mathrm{x}, \theta)$ for all $\theta \in \Theta$ 
3. Initialize $\left(\mathrm{E}_{1, \text { old }}, \mathrm{E}_{2, \text { old }}\right)=\left(\mathrm{E}_{1,0}, \mathrm{E}_{2,0}\right)$, where $\mathrm{E}_{1,0}$ and $\mathrm{E}_{2,0}$ are calculated from the experiments' data given in [13]

4. Fix $\left(E_{1 \text {, old }}, E_{2 \text {, old }}\right)$, using the EM algorithm to get estimation $f_{a \text {, new }}$

5. Fix $f_{a, \text { new }}$, using the EM algorithm to get estimation $\left(E_{1, \text { new }}, E_{2, \text { new }}\right)$

6. If $\left(\mathrm{E}_{1, \text { old }}, \mathrm{E}_{2 \text {, old }}\right) \neq\left(\mathrm{E}_{1, \text { new }}, \mathrm{E}_{2, \text { new }}\right)$, set $\left(\mathrm{E}_{1, \text { old }}, \mathrm{E}_{2, \text { old }}\right)=\left(\mathrm{E}_{1, \text { new }}, \mathrm{E}_{2, \text { new }}\right)$, go to step 4, otherwise, return $\left(E_{1}, E_{2}\right)=\left(E_{1, \text { new }}, E_{2, \text { new }}\right), f_{a}=f_{a \text {, new }}$

7. $\mathrm{t}=\mathrm{t}+1$, if $\mathrm{t}<\mathrm{nt}$, go to step 2, otherwise, stop.

where $n t$ is the number of time steps.

\section{$4 \quad$ Results}

We did experiments for two normal hearts and two abnormal hearts with right ventricular hypertrophy. The blood pressures were set to average clinical measurements as shown in Table 1. The procedure from the end of diastole to the end of systole was divided into 4 time intervals. Time 1 corresponds to the end of diastole and time 5 corresponds to the end of systole. The Young's modulus in each time interval were set initially as shown in Table 2, which is computed from the data given in [13].

Table 1. Blood Pressures in left and right ventricles

\begin{tabular}{|c|c|c|}
\hline & LV Blood Pressure & RV Blood Pressure \\
\hline Normal Heart & $120 \mathrm{mmHg}$ & $30 \mathrm{mmHg}$ \\
\hline RVH Heart & $120 \mathrm{mmHg}$ & $80 \mathrm{mmHg}$ \\
\hline
\end{tabular}

Table 2. Initial Young's modulus in each time interval

\begin{tabular}{|l|c|c|c|c|c|}
\hline & Time Interval & $1-2$ & $2-3$ & $3-4$ & $4-5$ \\
\hline \multirow{2}{*}{ Normal } & $\mathrm{E}_{1}(\mathrm{~Pa})$ & $50,000.0$ & $60,000.0$ & $70,000.0$ & $80,000.0$ \\
\cline { 2 - 6 } & $\mathrm{E}_{2}(\mathrm{~Pa})$ & $15,000.0$ & $18,000.0$ & $20,000.0$ & $22,000.0$ \\
\hline \multirow{2}{*}{ Abnormal } & $\mathrm{E}_{1}(\mathrm{~Pa})$ & $60,000.0$ & $70,000.0$ & $80,000.0$ & $90,000.0$ \\
\cline { 2 - 6 } & $\mathrm{E}_{2}(\mathrm{~Pa})$ & $18,000.0$ & $20,000.0$ & $22,000.0$ & $25,000.0$ \\
\hline
\end{tabular}

Using the statistical method, we get the estimation of Young's modulus in each time interval as shown in Table 3:

Table 3. Final estimation of Young's modulus in each time interval

\begin{tabular}{|l|c|c|c|c|c|}
\hline & Time Interval & $1-2$ & $2-3$ & $3-4$ & $4-5$ \\
\hline \multirow{2}{*}{ Normal } & $\mathrm{E}_{1}(\mathrm{~Pa})$ & $48,320.0$ & $59,780.0$ & $71,230.0$ & $77,590.0$ \\
\cline { 2 - 6 } & $\mathrm{E}_{2}(\mathrm{~Pa})$ & $14,210.0$ & $16,700.0$ & $20,980.0$ & $20,810.0$ \\
\hline \multirow{2}{*}{ Abnormal } & $\mathrm{E}_{1}(\mathrm{~Pa})$ & $61,700.0$ & $73,560.0$ & $82,370.0$ & $95,770.0$ \\
\cline { 2 - 6 } & $\mathrm{E}_{2}(\mathrm{~Pa})$ & $23,550.0$ & $24,350.0$ & $33,360.0$ & $36,790.0$ \\
\hline
\end{tabular}

The largest principal strain and stress in the free wall are shown in Fig. 4 and Fig. 5 respectively. From the figures, we know that the largest principal strain and stress get larger from the end of diastole to the end of systole in both normal and abnormal heart. Both normal heart and abnormal heart have larger strain and stress in the apex than in the base. Quantitatively, normal heart has smoother distribution of strain and stress than abnormal heart in the free wall. In addition, normal heart has larger strain 
than the abnormal heart although the stress has no much difference between normal heart and abnormal heart. This means the normal heart deforms more than the abnormal heart, but their stresses are similar because the normal heart has smaller Young's modulus.

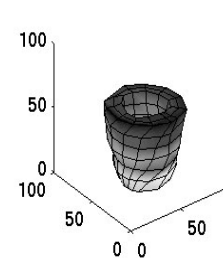

Time 1-2

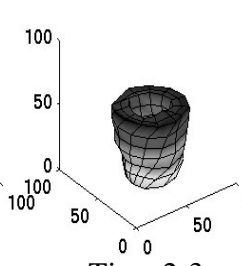

Time 2-3

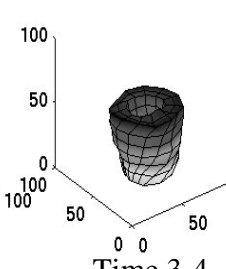

Time 3-4

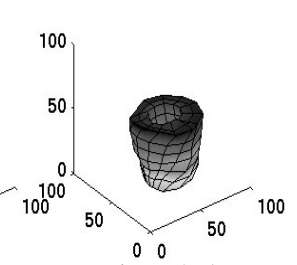

Time 4-5

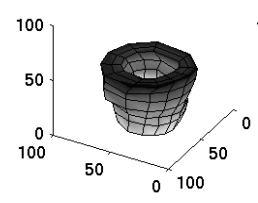

Time 1-2

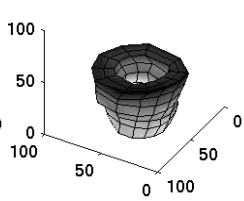

Time 2-3 (a)
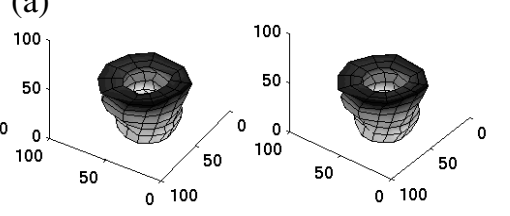

Time 4-5

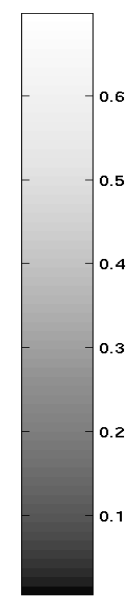

(b)

Fig. 4. Largest principal strain of free wall in 4 time intervals: (a) Left ventricle of normal heart (b) Left ventricle of abnormal heart with right ventricle hypertrophy

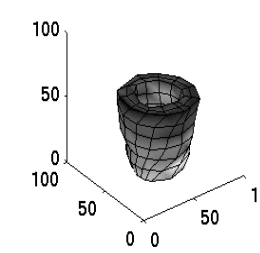

Time 1-2

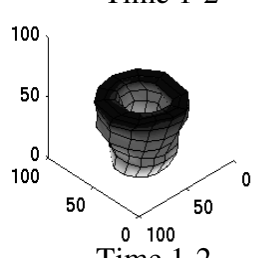

Time 1-2

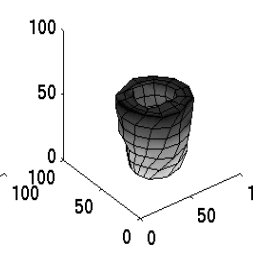

Time 2-3

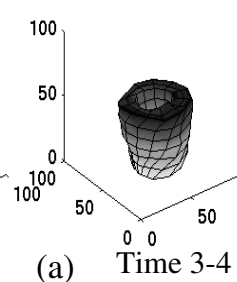

(a) Time 3-4

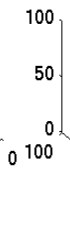

$0 \quad 100$

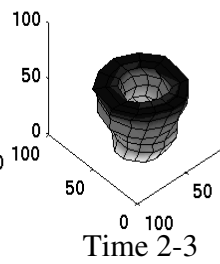

(b)

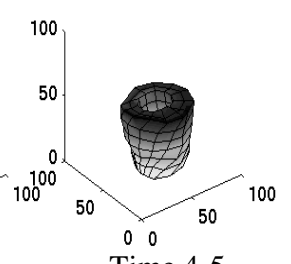

Time 4-5

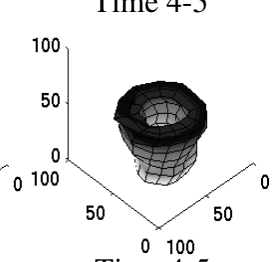

Time 4-5

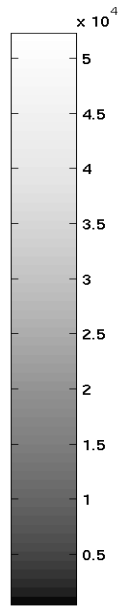

Fig. 5. Largest principal stress of free wall in 4 time intervals: (a) Left ventricle of normal heart (b) Left ventricle of abnormal heart with right ventricle hypertrophy

Comparisons between septum and free wall are also made as shown in Fig. 6 and Fig. 7. It shows that the normal heart has larger stress in the septum than in the free wall. For the abnormal heart, it has no much difference between septum and free wall. This is because the higher pressure in the right ventricle prevents the left ventricle of abnormal heart deforming as much as that of normal heart. 


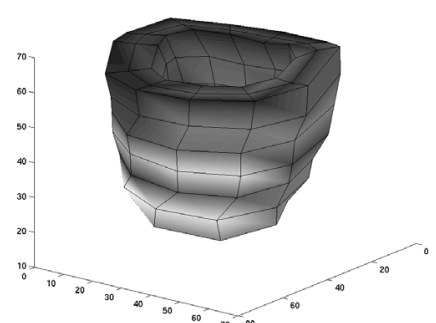

(a)

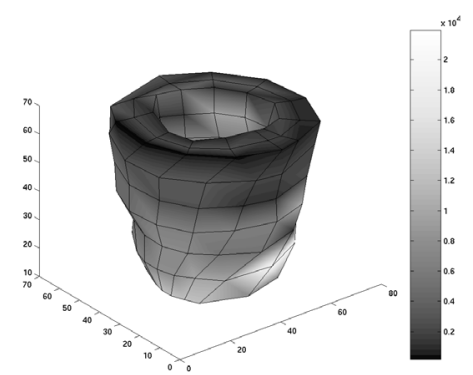

(b)

Fig. 6. Largest principal stress of normal heart: (a) Septum (b) Free wall in time interval 1-2

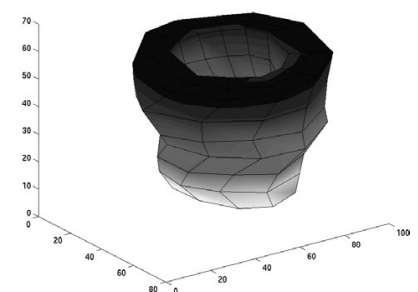

(a)

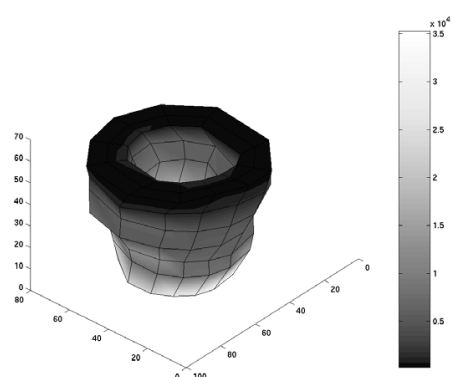

(b)

Fig. 7. Largest principal stress of left ventricle of abnormal heart: (a) Septum (b) Free wall in time interval 1-2

\section{Conclusions}

In-vivo strain and stress estimation is an intrinsically difficult problem. We developed a novel statistical method to compute the strain and stress by using accurate displacements reconstructed from MRI-SPAMM tagging and deformable model. Some interesting differences were found between abnormal hearts' left ventricle and normal hearts' left ventricle. The difference between septum and free wall were also found in the same left ventricle. The results may be used in the future clinical practice. More human hearts need to be studied to validate our model and our next goal is to compute strain and stress in right ventricle as well as left ventricle.

\section{Acknowledgements}

This research has been funded by grants from the NIH.

\section{References}

1. L. Glass, P. Hunter, A. McCulloch. Theory of Heart: Biomechanics, Biophysics, and Nonlinear Dynamics of Cardiac Function. Springer-Verlag, 1991.

2. K. Costa. The Structural Basis of Three-Dimensional Ventricular Mechanics, Ph.D. Dissertation, University of California, San Diego, CA, 1996. 
3. L. Axel, L. Dougherty. Heart wall motion: Improved method of spatial modulation of magnetization for MR imaging. Radiology, 272:349-50, 1989.

4. D. N. Metaxas. Physics-based deformable models: applications to computer vision, graphics, and medical imaging. Kluwer Academic Publishers, Cambridge, 1996.

5. X. Papademetris, A. Sinusas, D. P. Dione, J. S. Duncan. Estimation of 3D left ventricular deformation from echocardiography. Medical Image Analysis, 5:17-28, 2001.

6. M. Sermesant, Y. Coudiere, H. Delingette, N. Ayache, J. A. Desideri. An ElectroMechanical Model of the Heart for Cardiac Image Analysis. In Medical Image Computing and Computer-Assisted Intervention (MICCAI'01), 2001.

7. Y. C. Fung. Biomechanics: mechanical properties of living tissues, 2nd ed. SpringerVerlag, 1993.

8. M. W. Hyer. Stress Analysis of Fiber-Reinforced Composite Materials. McGraw-Hill, 1998.

9. A. Amini, Y. Chen, R. W. Curwen, V. Manu, J. Sun. Coupled B-Snake grides and constrained thin-plate splines for analysis of 2D tissue deformations from tagged MRI. IEEE Transaction on Medical Imaging 17(3),344-356, 1998.

10. D. D. Streeter Jr., W. T. Hanna. Engineering mechanics for successive states in canine left ventricular myocardium: I. Cavity and wall geometry. Circulation Research, 33:639-655, 1973.

11. K. Bathe. Finite element procedures in engineering analysis. Prentice Hall, 1982.

12. O. C. Zienkiewicz, R. L. Taylor. The finite element method. McGraw-Hill, 1989.

13. T. P. Usyk, R. Mazhari, A. D. McCulloch. Effect of laminar orthotropic myofiber architecture on regional stress and strain in the canine left ventricle. Journal of Elasticity, 61, 2000.

14. Haber, D. N. Metaxas, L. Axel. Three-dimensional motion reconstruction and analysis of the right ventricle using tagged MRI. Medical Image Analysis, 4, 2000.

15. P. J. Bickel, K. A. Doksum. Mathematical statistics: basic ideas and selected topics, Vol. I. Prentice Hall, 2001.

16. Y. C. Fung. A first course in continuum mechanics: for physical and biological engineers and scientists, 3rd ed. Prentice Hall, 1994.

17. J. G. Pinto, Y. C. Fung. Mechanical properties of the heart muscle in the passive state. Journal of Biomechanics, 6:597-616,1973.

18. Y. C. Pao, G. K. Nagendra, R. Padiyar, E. L. Ritman. Derivation of myocardial fiber stiffness equation on theory of laminated composite. Journal of Biomechanical Engineering, 102:252-257, 1980.

19. L. L. Demer, F.C.P. Yin. Passive biaxial properties of isolated canine myocardium. Journal of Physiology, 339:615-630, 1983.

20. F.C.P. Yin, R. K. Strumpf, P.H. Chew, S.L. Zeger. Quantification of the mechanical properties of non-contracting myocardium. Journal of Biomechanics, 20:577-589, 1987.

21. J.D. Humphrey, F.C.P. Yin. Biomechanical experiments on excised myocardium: Theoretical considerations. Journal of Biomechanics, 22:377-383, 1989.

22. J.D. Humphery, F.C.P. Yin. On constitutive relations and finite deformations of passive cardiac tissue: I. A pseudostrain-energy function. Journal of Biomechanical Engineering, 109:298-304, 1987.

23. C. Guyton, J. E. Hall. Textbook of Medical Physiology, 10th ed. W.B. Sauders Company, 2000.

24. X. Papademetris. Estimation of 3D left ventricular deformation from medical images using biomechanical models, Ph.D. Dissertation, Yale University, New Haven, CT, May 2000.

25. Haber. Three dimensional motion reconstruction and analysis of the right ventricle from planar tagged MRI. Ph.D. Dissertation, University of Pennsylvania, Philadelphia, PA, 2000.

26. Haber, D. Metaxas, L. Axel. Motion analysis of the right ventricle from MRI images. In Medical Image Computing and Computer-Assisted Intervention (MICCAI'98), 1998. 